\title{
Ecotoxicity of heat-treated Kapur and Japanese larch
}

\begin{abstract}
Kapur (Dryobalanops sp.) and Japanese larch (Larix kaempferi) wood samples were heattreated in air at $180{ }^{\circ} \mathrm{C}$ for 24 and $48 \mathrm{~h}$. Leaching and toxicity tests were conducted to determine the ecotoxicity effect of these heat-treated woods on the aquatic environment due to heat treatment. The toxicity of conventional preservative-treated woods, i.e. chromated copper arsenate (CCA) and ammoniacal copper quat (ACQ), was also determined for comparison purposes. Acute toxicity tests were performed using two aquatic organisms, Daphnia magna and Vibrio fischeri according to the Organization for Economic Co-operation and Development standard procedures and Microtox assay, respectively. Significantly low toxicity to D. magna was found for the heat-treated Kapur compared to that of untreated Kapur, while heat-treated Japanese larch did not show any toxicity effect. As expected, ACQtreated samples showed the highest toxicity to D. magna, followed by that of CCA, though toxicity of both preservative-treated woods further reduced over time. Hence, heat treatment of Kapur and Japanese larch were believed to be not harmful to the aquatic ecosystem.
\end{abstract}

Keyword: Kapur; Japanese larch; Heat treatment; Toxicity; Preservative-treated wood; Aquatic ecosystems 